\title{
Not even wrong: Comment by Wagg et al.
}

Wagg, Cameron ; Barry, Kathryn E ; O’Brien, Michael J ; McKenzie-Gopsill, Andrew ; Roscher, Christiane ; Eisenhauer, Nico ; Schmid, Bernhard

DOI: https://doi.org/10.1002/ecy.2805

Posted at the Zurich Open Repository and Archive, University of Zurich ZORA URL: https://doi.org/10.5167/uzh-180660

Journal Article

Accepted Version

Originally published at:

Wagg, Cameron; Barry, Kathryn E; O’Brien, Michael J; McKenzie-Gopsill, Andrew; Roscher, Christiane; Eisenhauer, Nico; Schmid, Bernhard (2019). Not even wrong: Comment by Wagg et al. Ecology, 100(10):e02805.

DOI: https://doi.org/10.1002/ecy.2805 


\section{Not even wrong: Comment by Wagg et al.}

3 Cameron Wagg ${ }^{1}$, Kathryn E. Barry ${ }^{2,3}$, Michael J. O’Brien ${ }^{4}$, Andrew McKenzie-Gopsill',

4 Christiane Roscher ${ }^{3,6}$, Nico Eisenhauer ${ }^{3,7}$, Bernhard Schmid $^{8 *}$

\section{5}

$6 \quad{ }^{1}$ Fredericton Research and Development Center, Agriculture and Agri-Food Canada, 850

7 Lincoln Road, Fredericton, New Brunswick, E3B 4Z7, Canada

$8 \quad{ }^{2}$ Systematic Botany and Functional Biodiversity, Institute of Biology, Leipzig University;

9 Johannisallee 21, 04103 Leipzig, Germany

$10{ }^{3}$ German Centre for Integrative Biodiversity Research (iDiv) Halle-Jena-Leipzig; Deutscher

11 Platz 5e, 04103 Leipzig, Germany.

$12{ }^{4}$ Área de Biodiversidad y Conservación, Universidad Rey Juan Carlos, c/Tulipán s/n, E-

1328933 Móstoles, Spain

$14{ }^{5}$ Charlottetown Research and Development Center, Agriculture and Agri-Food Canada, 440

15 University Ave., Charlottetown, Prince Edward Island, C1A 7Z5, Canada

$16{ }^{6}$ Department of Physiological Diversity, UFZ - Helmholtz Centre for Environmental

17 Research, Permoserstr. 15, Leipzig 04318, Germany

$18{ }^{7}$ Leipzig University, Institute of Biology, Deutscher Platz 5e, 04103 Leipzig, Germany

$19{ }^{8}$ Department of Geography, University of Zürich, Winterthurerstrasse 190, 8057, Zürich,

20 Switzerland

21

$22 *$ Author for correspondence 


\section{Main text}

A recent paper by Pillai and Gouhier (2019) (PG) in Ecology argues that biodiversityecosystem functioning (BEF) calculations of biodiversity effects using the additive partitioning approach introduced by Loreau and Hector (2001) (LH) are fundamentally flawed. PG claim that the null expectation of BEF research, and thus of the additive partitioning approach, stating that in the absence of biodiversity effects the performance of a mixture should equal the average performance of its component species' monocultures, is an extension of the neutral theory of species coexistence (which strictly speaking would require equal monoculture yields not required by LH). Further, they argue that a more reasonable null expectation based on coexistence theory (CT) would be "for species in mixtures to coexist and by some form of niche partitioning overyield." Based on these assertions, PG conclude that "overyielding of ecosystem properties should be a natural outcome of coexistence" and thus past BEF research has overestimated positive effects of biodiversity, for which this natural outcome could not be considered. Although BEF and CT share related ecological concepts, their different goals, origins, and intended uses prevent us from directly substituting their applications and implications. We provide a brief background on some fundamental concepts that underpin BEF research to elucidate potential misconceptions that may have led PG to their conclusion that, based on $\mathrm{CT}$, the null expectation of BEF research used by LH is flawed. Despite the differences between BEF and CT research origins and approaches, their commonalities have been recognized early on (Harper 1977, Vandermeer 1981, Loreau 2004) and require further empirical investigation that may lead to novel opportunities to theoretically predict, and empirically measure, how coexistence mechanisms may contribute to observed effects of biodiversity on ecosystem functioning in nature. PG claim that findings of enhanced ecosystem functioning in more biodiverse systems based on the additive partitioning approach introduced by LH are inflated due to the 
assumption of neutrality between species. They claim that the null expectation of no effect on ecosystem functioning with increasing species richness, while holding community density constant, is flawed because as richness increases these species must coexist by occupying different niches. Here we argue that this assertion may not apply for three reasons:

1. The history of BEF research and null expectations of no effect of additional species on ecosystem function has evolved from agricultural science and plant population biology independently of CT.

2. Although long-term stable coexistence may often be associated with overyielding, there can be coexistence without overyielding.

3. There can be overyielding without stable coexistence (Turnbull et al. 2013), and it is possible to create short-term communities of higher richness and productivity than possible in the long term (Pfisterer et al. 2004).

\section{BEF research evolved independently of CT and thus is subject to different null} expectations

BEF research started with the simple question about potential effects of species loss from an ecosystem on its functioning, often measured as biomass yield. For this, plant species richness was experimentally manipulated by holding total sowing or planting density constant to avoid confounding species richness and community density (substitutive as opposed to additive design; Schmid et al. 2002a). These experiments commonly found that plant species richness-yield relationships were positive but with declining increases as more and more species were assembled (e.g. Tilman et al. 1996, Hector et al. 1999; meta-analysis in Balvanera et al. 2006). This generated some debate at the time as to whether these positive effects of plant diversity on productivity were simply due to the increasing probability of including one, or a few, highly productive species in more diverse systems (e.g., Huston 
74 1997, Wardle 2016, Eisenhauer et al. 2016). Note that this requires the so-called rule of

75 constant final yield (the first law of plant population biology according to Harper 1977),

76 which allows these highly productive species to approach the same biomass in mixtures as in

77 monoculture, even though they are sown at lower density in mixture. The initial skepticism

78 against the possible existence of positive BEF relationships was in part related to theory

79 which predicted negative relationships between ecosystem complexity and stability (May

80 1972). A further reason of skepticism were the expectations of early agricultural botanists

81 that, at least under ideal conditions, the best-performing monoculture should always be more

82 productive than the best mixture (no transgressive overyielding of mixtures, Harper 1977).

83 Assuming that BEF relationships must be positive by definition due to CT (as understood by

84 PG) is thus counter to these historical expectations.

85 This research was based, to a large extent, on the pioneering work of de Wit (1960, de

86 Wit et al 1966). De Wit introduced the replacement series experimental design, where total

87 density is kept constant but the mixing ratio of two species is varied. Monocultures of species

88 are used as a reference for identifying the optimal sward-mixing ratio along a replacement

89 series. This design is based on the null expectation that if there are no effects of mixing,

90 individuals of different species are competitively equivalent and thus mixtures should yield

91 as predicted by adding the monocultures in the proportions given by the frequency of the

92 species in mixture. Thus, classic replacement series assess the effect of increasing the sown

93 density of a species ' $i$ ', while equally reducing the sown density of a species ' $j$ ' to maintain a

94 constant sowing density (Fig. 1a). If the null expectation is that the yield per individual of a

95 species is constant in constant community density, then a species contribution to yield is its

96 monoculture yield weighted by its sown density proportion (Fig. 1b). The sum of the species

97 relative yields is the relative yield total $(R Y T)$, and if species are in mixture with a constant

98 community density, then the null hypothesis is that the $R Y T=1$. According to this first 
scenario in Fig. 1, if all species were identical and density constant between mixtures and monocultures, then increasing species richness should have no effect on yield and no overyielding should occur $(R Y T=1$ for all species richness levels). ecological mechanisms can be at play. Some of these ecological mechanisms may be related to $\mathrm{CT}$ and the limiting-similarity and competitive-exclusion hypotheses (MacArthur \& Levins 1967, Chesson 2000, Weiner and Freckleton 2010, Barabás et al. 2018). For instance, by reducing the density of species $i$ in mixture, it may become released from intraspecific competition that could lead to increased yield per individual of species $i$. If the other species $j$ does not suffer more from $i$ than $i$ gains from $j$, or if even both species behave in the described manner in mixture, i.e. if overall intraspecific competition is stronger than

110 interspecific competition, then $R Y T>1$ (Fig. 1: scenarios two and three). As long as there is

111 no facilitation between the two species, a maximum $R Y T=2$ can be achieved in two-species

112 mixtures according to the rule of constant final yield (Fig. 1: scenario two; Schmid et al.

113 2002b). If one or both species facilitate(s) the growth of the other - such as in the case of

114 legumes facilitating neighboring species (Spehn et al. 2005, Temperton et al. 2007), and if

115 there would be no interspecific competition at all, then $R Y T>2$ would theoretically be 116 possible.

117 Whatever the species richness, according to scenario two the expected mixture yield

118 is the sum of all the component species' monoculture yields. Such a situation with no niche

119 overlap and no interspecific competition between species is of course highly unrealistic but 120 important to consider in the context of PG's new null expectation for biodiversity effects,

121 because it reflects this null expectation in its most extreme form. They argue that the null 122 expectation of BEF research should be that mixtures are overyielding to the degree to which 123 species niches do not overlap and thus do not compete for the same resources. In this case, 
124 niche overlap between species under the null expectation could be measured via $R Y T$ and, by

125 doing this in all possible two-species mixtures, null expectations for mixtures of more than

126 two species could be derived. However, to tease apart the two-way interactions from the

127 higher-order interactions would require new experiments that include replicate monocultures,

128 replicate 2-species mixtures with all pairs of species and replicated mixtures with higher

129 levels of diversity, a heroic effort that has not been done thus far. Not surprisingly, the real

130 biodiversity effect of interest calculated by PG is generally negative, because they use no

131 correction for multiple niche overlap in mixtures of three or more species. Their null

132 expectation is thus fundamentally different from the common null expectation used in LH,

133 which corresponds to the first scenario in Fig. 1. Here the expected mixture yield is not the

134 sum but the average of all the component species' monoculture yields and thus increasing

135 species richness does not change yield.

136 The additive partitioning of biodiversity effects introduced by LH allowed for the first

137 time to quantify different ways in which relative yields of species could combine to total

138 yield, something that previously was done in more qualitative ways e.g. by the replacement-

139 series approach of de Wit (1960). Additive partitioning works with relative yields to separate

140 the net biodiversity effect (difference between mixture yield and the average of monoculture

141 yields) into two additive components called the 'complementarity effect' (CE) and the

142 'selection effect' (SE). The additive partitioning was developed to assess early BEF debates

143 as to whether positive net effects $(\mathrm{NE}=\mathrm{CE}+\mathrm{SE})$ of biodiversity were driven more or less by

144 particularly productive species (SE) or by a generally increased species performance in

145 mixture (CE). It was not intended to tease apart the density-dependent competitive effects, or

146 fitness differences, between species pairs used in CT, or to provide direct evidence of any one

147 particular ecological mechanism underpinning BEF relationships as PG incorrectly assume. It

148 was already known prior to the development of the additive partitioning that the use of 
149 relative yields confounds intraspecific and interspecific density effects (see Connolly 1986,

150 Jolliffe 2000).

151 Application of the LH additive partitioning scheme increases the utility of

152 replacement series results. Originally, the aim of the RYT was to compare species of similar

153 monoculture performance in a replacement series. This similarity in monoculture yield

154 simplifies the interpretation of $R Y T>1$, because if species monocultures are similar in yield,

155 a $R Y T>1$ will always require a contribution of both species. In this case, the overyielding of

156 the mixture is mostly or fully due to the CE from the additive partitioning method. However,

157 if a more productive species is mixed with a less productive species, $R Y T>1$ can but does

158 not have to be achieved with the SE alone. It is here where the usefulness of the additive

159 partitioning approach can be seen: it distinguishes this case by assigning the NE of $R Y T>1$

160 to SE, where the mixture becomes essentially a monoculture of the more productive species,

161 from other cases where both species contribute to the biodiversity effect. In fact, if two

162 species differ in monoculture yields, there is a large range of possible contributions of SE and

163 CE that lead to NE $>0$ and $R Y T>1$ (see Fig. 1). Similar arguments apply to cases with $R Y T$

$164<1$ (Loreau and Hector 2001).

165 Considering overyielding in species mixtures originates from assessing the optimal

166 sward mixing in managed grasslands and whether it is better to plant monocultures or

167 mixtures (e.g. de Wit 1960). The use of the additive partitioning of biodiversity effects to

168 tease apart the relative contributions of species in mixtures as well as to explain why mixtures

169 perform differently than the average monoculture is useful for the analysis of BEF

170 experiments. The arguments of PG overlooked this rich history and the intended use for

171 assessing yield that does not need to be directly connected to the long-term coexistence of

172 species. A further very useful aspect of the additive partitioning method is that it applies not

173 only to two-species mixtures but also to any other species richness levels. The only 
requirement is that species yields can be separated in mixtures and that all species can also be 175 grown in monoculture.

176

177 2. There can be coexistence without overyielding

178 The fact that CEs and SEs do not have a direct mechanistic explanation in terms of

179 coexistence or niche theory may be one of the frustrations of PG. Indeed, a common

180 misconception is that complementarity effects should be directly equivalent to resource

181 complementarity or spatial complementarity (Barry et al. 2019). While this is an interesting

182 hypothesis, it has received little empirical support so far. For example, in a forest experiment,

183 physical crown complementarity was actually more closely related to SEs than to CEs

184 (Williams et al. 2017), perhaps reflecting a competitive trait hierarchy (Kunstler et al. 2012).

185 Disconnects between species coexistence and the mechanisms by which species contribute to

186 overyielding reveal the difficulty in logically substituting the concepts of one into the other

187 and may be based on misconceptions of the origins and objectives of BEF research for the

188 following reasons.

189 First, although positive effects of biodiversity are commonly observed in both

190 experimental and natural contexts and across different ecosystems (Balvanera et al. 2006,

191 Duffy et al. 2017), the experimental mixing of species does not always result in overyielding.

192 In rare cases, antagonistic interactions among species have also been observed in biodiversity

193 experiments leading to mixtures having $R Y T<1$. This occurs if overall inter-specific

194 competition is stronger than intraspecific competition in a mixture (Fig. 1: scenario four). For

195 example, this has been observed in bacterial communities, where species increase the

196 production of allelopathic toxins in mixtures, thus leading to a strongly negative biodiversity

197 effect without the competitive exclusion of species during the course of an experiment

198 (Jousset et al. 2011, Becker et al. 2012). Other situations resulting in $R Y T<1$ can result from 
199 positive density-dependence, for example the lack of attracting mutualists by a species at

200 lower densities - such as in the case where the performance of a species is dependent on

201 positive plant-soil feedback effects (van der Putten et al. 2013). While in these cases both

202 species may suffer in a two-species mixture, $R Y T<1$ can also result in situations where an

203 unproductive species $j$ suppresses a more productive species $i$, even though $j$ may be less

204 suppressed by $i$ than by itself (Loreau \& Hector 2001).

205 Second, if similar species are expected to compete more strongly for common

206 resources, then it may also be expected that due to their similarity the species also have

207 relatively equal competitive effects on each other (Ebeling et al. 2014). Thus, in mixtures of

208 highly similar species, neither will have a large enough competitive advantage to exclude the

209 other due to their similar fitness (Aarssen 1983, Hubbell 2001, Adler et al. 2007, Mayfield

210 and Levine 2010). The consequence of species being competitively equivalent with a high

211 level of niche overlap could again result in species coexisting without overyielding. In such

212 cases, it is clear that statements such as "overyielding of ecosystem properties should be a

213 natural outcome of coexistence" by PG have overlooked the fact that species in mixture can

214 interact and coexist in a way that does not result in overyielding.

215

216 3. There can be overyielding without stable coexistence

217 The hypothesized mechanisms that underpin the empirical observations of positive

218 BEF relationships and the hypothesized drivers of coexistence run parallel to each other

219 (Loreau 2004, Loreau 2010, Caroll et al. 2011, Turnbull et al. 2013, Turnbull et al. 2016).

220 Such parallels may give the impression that the approaches are aimed at achieving the same

221 objective and may be easily intermixed as done by PG. However, CT and BEF relationships

222 are not completely comparable, and long-term stable coexistence is not a necessary condition

223 for enhanced ecosystem functioning in more diverse systems as implied by PG. 
First, CT and the additive partitioning method are used at two different temporal

scales. Additive partitioning is applied to a state variable usually within a single growing

season. In contrast, classic coexistence models are based on per capita growth rates modeled

over many demographic turnover events in a community at stable equilibrium and are

therefore process variables. Commonalities between a complementarity effect in BEF

research and competitive interactions from CT may be more easily drawn in biodiversity

experiments involving trees, where the yearly growth of individuals can be followed (Huang

et al. 2018), or in experiments involving perennial herbaceous plants if we replace 'per capita

growth rate' with 'species biomass accumulation rate' within a single growing season, and if

we also assume that the species monoculture yield is an indication of species carrying

capacity $K$. The use of monoculture yields to infer $K$ is not unreasonable because according

to the law of constant final yield (monocultures sown at a very high density undergo 'self-

thinning' until the constant final yield is met, Harper 1977, Weiner and Freckleton 2010).

This constant final yield can also be reached with much lower density, where individual

plants grow to be larger (Harper 1977). The concept of constant final yield in monocultures

may be considered akin to the concept of a species carrying capacity $(K)$ used in species

competition-coexistence frameworks (Westoby 1981). Thus, plants may 'adjust' their 'per

capita growth rate' within a single cohort to compensate for density variations to achieve the

constant final yield $(K)$. This is an observed phenomenon in various plant species

243 populations, where individual plants at lower intraspecific densities grow larger, likely due to

244 greater resource availability per individual (Bazzaz and Harper 1976, Malmberg and Smith

245 1982, Roscher and Schumacher 2016). However, effects of species richness on thinning and

246 constant final yield in mixtures have barely been explored and are complicated (He et al.

247 2005, Roscher et al. 2007), further demonstrating the complexity of relating BEF research to

248 CT. 
250 (Cardinale et al. 2007, Marquard et al. 2009, Reich et al. 2012, Huang et al. 2018). Temporal

251 changes in CEs (and RYT) can be generated from coexistence models (Turnbull et al. 2013).

252 However, these theoretical models demonstrate that enhanced ecosystem functioning can

253 occur even when long-term stable coexistence is not possible. Turnbull et al. (2013) found

254 that communities can overyield even when stabilizing niche differences (sensu Chesson

255 2000) cannot overcome fitness differences and therefore allow for stable coexistence. This

256 'transient' overyielding may occur frequently in natural communities, where environmental

257 conditions change and may lead to long-term unstable persistence rather than long-term

258 stable coexistence (Wagg et al. 2017). Furthermore, nutrient addition sometimes can increase

259 overyielding and simultaneously decrease the number of species that are able to coexist,

260 indicating that enhanced ecosystem functioning with increased species richness and

261 coexistence in these systems may be driven by different mechanisms (Fox and Harpole 2008,

262 Harpole et al. 2016). Therefore, the application of the coexistence models to diverse

263 perennial grasslands, where much of the BEF research originated, including the use of the

264 additive partition method, is extremely challenging as species vary in their demographic

265 turnover rates. There is no constant per capita growth rate considering that plant yield-

266 density relationships follow the low of constant final yield, and that plant communities can be

267 highly diverse (more than species pairs), resulting in complex higher-order interactions that

268 are difficult to parameterize.

\section{Conclusions}

271 Coexistence mechanisms based on niche partitioning and fitness differences are

272 important ecological mechanisms that can relate to positive effects of biodiversity and are

273 useful for deriving empirically testable hypotheses for future studies on the mechanisms 
underlying BEF relationships. However, the proposed redefining of the null hypothesis by

275 PG, claiming that coexistence always implies "trivial" positive biodiversity effects against

276 which "true" biodiversity effects would have to be evaluated is circular in its own way as

277 indicated by the use of the trivial component of biodiversity effects as a measure of

278 coexistence. In other words, this null hypothesis defines a biodiversity effect as proof of no

279 effect. Furthermore, the use of classic coexistence models for predicting species competitive

280 outcomes at higher levels of diversity (with more than two species) is limited due to higher-

281 order interactions that become more complex across increasing levels of species richness

282 (Levine et al. 2017, Barabás et al. 2018). The additive partitioning of biodiversity effects is

283 also not theoretically limited by first having to parameterize all species' pairwise and higher

284 order interactions in diverse plant mixtures. While the use of CT indeed provides many

285 insights for understanding ecological mechanisms that support biodiversity in nature, its use

286 in practical and applied settings, such as in agricultural ecosystems, where species mixtures

287 are sown at predefined densities and responses are quantified within a single growing season,

288 may not be as useful as relative yields and the additive partitioning method.

289

\section{$290 \quad$ References}

291 Aarssen, L.W. 1983. Ecological combining ability and competitive combing ability in plants:

292 toward a general evolutionary theory of coexistence in systems of competition. The

$293 \quad$ American Naturalist 122:707-731.

294 Adler, P., J. HilleRisLambers and J.M. Levine. 2007. A niche for neutrality. Ecology Letters

$295 \quad 10: 95-104$.

296 Balvanera, P., A.B. Pfisterer, N. Buchmann, J.S. He, T. Nakashizuka, D. Raffaelli and B.

297 Schmid. 2006. Quantifying the evidence for biodiversity effects on ecosystem

298 functioning and services. Ecology Letters 9:1146-1156. 
299 Barabás, G., R.D. Andrea and S.M. Stump. 2018. Chesson's coexistence theory. Ecological

$300 \quad$ Monographs 88:277-303.

301 Barry, K.E., et al. 2019. The future of complementarity: disentangling causes from

302 consequences. Trends in Ecology and Evolution 34:167-180.

303 Bazzaz, F.A. and J.L. Harper. 1976. Relationship between plant weight and numberss in 304 mixed populations of Sinapsis alba (L.) Rabenh. and Lepidium sativum L. Journal of $305 \quad$ Applied Ecology 13:211-216.

306 Becker, J., N. Eisenhauer, S. Scheu and A. Jousset. 2012. Increasing antagonistic interactions 307 cause bacterial communities to collapse at high diversity. Ecology Letters 15:486-474.

308 Cardinale, B. J., J. P. Wright, M. C. Cadotte, I. T. Carroll, A. Hector, D. S. Srivastava, M.

309 Loreau and J. J. Weiss. 2007. Impacts of plant diversity on biomass increase through time

310 because of species complementarity. Proceedings of the National Academy of Sciences,

$311 \quad$ USA 104:18123-18128.

312 Carroll, I.T., B.J. Cardinale and R.M. Nisbet. 2011. Niche and fitness differences relate the 313 maintenance of diversity to ecosystem functioning. Ecology 92:1157-1165.

314 Chesson, P. 2000. Mechanisms of maintenance of species diversity. Annual Review of 315 Ecology, Evolution, and Systematics, 31, 343-366.

316 Connolly, J. 1986. On difficulties with the replacement-series methodology in mixture 317 experiments. Journal of Applied Ecology 23:125-137.

318 de Wit, C. T. 1960. On competition. Verslag Landbouwkundige Onderzoek 66.8:1-82.

319 de Wit, C. T., P. G. Tow, and G. C. Ennik. 1966. Copetition between legumes and grasses. $320 \quad$ Verslag Landbouwkundige Onderzoek 687:1-30.

321 Harpole, W.S. and J.W. Fox. 2008. Revealing how species loss affects ecosystem function: 322 the trait-based Price equation partition. Ecology 89:269-279. 
323 Duffy, J.E., C.M. Goodin and B.J. Cardinale. 2017. Biodiversity effects in the wild are

324 common and as strong as key drivers of productivity. Nature 549:261-264.

325 Ebeling, A. Et al. 2014. A trait-based experimental approach to understand the mechanisms

326 underlying biodiversity-ecosystem functioning relationships. Basic and Applied

327 Ecology 15: 229-240.

328 Eisenhauer, N., D. B. Barnes, S. Cesarz, D. Craven, O. Ferlian, F. Gottschall, J. Hines, A.

329 Sendek, J. Siebert, M. P. Thakur and M. Türke. 2016. Biodiversity-ecosystem function

330 experiments reveal the mechanisms underlying the consequences of biodiversity

331 change in real world ecosystems. Journal of Vegetation Science 27:1061-1070.

332 Harper, J.L. 1977. Population Biology of Plants. Academic Press, London.

333 Harpole, W.S., et al. 2016. Addition of multiple limiting resources reduces grassland

334 diversity. Nature 537:93-96.

335 He, J.-S., K.S. Wolfe-Bellin, B. Schmid and F.A. Bazzaz. 2005. Density may alter diversity-

336 productivity relationships in experimental plant communities. Basic and Applied

$337 \quad$ Ecology 6:505-517.

338 Hector, A., et al. 1999. Plant diversity and productivity experiments in European grasslands.

339 Science 286:1123-1127.

340 Huang, Y., et al. 2018. Impacts of species richness on productivity in a large-scale

341 subtropical forest experiment. Science 362:80-83.

342 Hubbell, S.P. 2001. The unified neutral theory of biodiversity and biogeography (MBP-32).

$343 \quad$ Princeton University Press.

344 Huston, M.A. 1997. Hidden treatments in ecological experiments: re-evaluating the

345 ecosystem function of biodiversity. Oecologia 110:449-460.

346 Jolliffe, P. A. 2000. The replacement series. Journal of Ecology 88:371-385. 
Jousset, A., B. Schmid and N. Eisenhauer. 2011. Genotypic richness and dissimilarity opposingly affect ecosystem functioning. Ecology Letters 14:537-545.

Kunstler, G., S. Lavergne, B. Courbaud, W. Thuiller, G. Vieilledent, N.E. Zimmermann, J. Kattge, D.A. Coomes. 2012. Competitive interactions between forest trees are driven

Loreau, M. 2004. Does functional redundancy exist? Oikos 104:606-611.

Loreau, M. 2010. Linking biodiversity and ecosystems: towards a unifying ecological theory. Philosophical Transactions of the Royal Scoiety B 365:49-60.

Loreau, M., and A. Hector. 2001. Partitioning selection and complementarity in biodiversity

MacArthur, R. H., and R. Levins. 1967. The limiting similarity, convergence, and divergence experiments. Nature 412:72 76 .

Malmberg, C. and H. Smith. 1982. Relationship between plant weight and density in mixed populations of Medicago sativa and Trifolium pretense. Oikos 38:365-368.

Marquard, E., A. Weigelt, V. M. Temperton, C. Roscher, J. Schumacher, N. Buchmann, M.

Fischer, W. W. Weisser and B. Schmid. 2009. Plant species richness and functional

May, R.M. 1972. Will a large complex system be stable? Nature 238:413-414.

369

Mayfield, M. and J. Levine. 2010. Opposing effects of competitive exclusion on the phylogenetic structure of communities. Ecology Letters 13:1085-1093. 
371 Pfisterer, A.B., J. Joshi, B. Schmid and M. Fischer. 2004. Rapid decay of diversity-

372 productivity relationships after invasion of experimental plant communities. Basic and $373 \quad$ Applied Ecology 5:5-14.

374 Pillai, P. and T.C. Gouhier. 2019. Not even wrong: the spurious measurement of

375 biodiversity's effects on ecosystem functioning. Ecology (in press)

$376 \quad$ https://doi.org/10.1002/ecy.2645

377 Reich, P.B., D. Tilman, F. Isabell, K. Mueller, S. E. Hobbie, D. F. B. Flynn and N.

378 Eisenhauer. 2012. Impacts of biodiversity loss escalate through time as redundancy fades.

$379 \quad$ Science 336:589-592.

380 Roscher, C. and J. Schumacher. 2016. Positive diversity effects on productivity in mixtures

381 of arable weed species as related to density-size relationships. Journal of Plant Ecology

$3829: 792-804$.

383 Roscher, C., J. Schumacher, W.W. Weisser, B. Schmid and E.D. Schulze. 2007. Detecting

384 the role of individual species for overyielding in experimental grassland communities

385 composed of potentially dominant species. Oecologia. 154:535-549.

386 Schmid, B., A. Hector, M.A. Huston, P. Inchausti, I. Nijs, P.W. Leadley and D. Tilman.

387 2002a. The design and analysis of biodiversity experiments. Pages 61-75 in M. Loreau,

388 S. Naeem and P. Inchausti, editors. Biodiversity and ecosystem functioning: synthesis

389 and perspectives. Oxford University Press, Oxford, UK.

390 Schmid, B., J. Joshi and F. Schläpfer. 2002b. Emperical evidence for biodiversity-ecosystem

391 functioning relationships. Pages $120-150$ in A. Kinzig, S. Pacala and D. Tilman,

392 editors. The Functional consequences of biodiversity: empirical progress and

393 theoretical extensions (MPB-33). Princeton University Press, New Jersey, USA.

394 Spehn, E.M., et al. 2005. Ecosystem effects of biodiversity manipulations in European

395 grasslands. Ecological Monographs 75:37-63. 
396 Temperton, V. M., P. N. Mwangi, M. Scherer-Lorenzen, B. Schmid, and N. Buchmann.

397 2007. Positive interactions between nitrogen-fixing legumes and four different

398 neighbouring species in a biodiversity experiment. Oecologia 151:190-205.

399 Tilman, D., D. Wedin, and J. Knops. 1996. Productivity and sustainability influenced by

$400 \quad$ biodiversity in grassland ecosystems. Nature 379:718-720.

401 Turnbull, L.A., J. M. Levine, M. Loreau and A. Hector. 2013. Coexistance, niches and

402 biodiversity effects on ecosystem functioning. Ecology Letters 16:116-127.

403 Turnbull, L.A., F. Isbell, D.W. Purves, M. Loreau and A. Hector. 2016. Understanding the

404 value of plant diversity and ecosystem functioning through niche theory. Proceedings

405 of the Royal Society B 283:20160536.

406 Vandermeer, J. 1981. The interference production principle: an ecological theory for

$407 \quad$ agriculture. BioScience 31:361-364.

408 van der Putten, W.H., et al. 2013. Plant-soil feedbacks: the past, the present and future

$409 \quad$ challenges. Journal of Ecology 101:265-276.

410 Wagg, C., M.J. O’Brien, A. Vogel, M. Scherer-Lorenzen, N. Eisenhauer, B. Schmid and A.

411 Weigelt. 2017. Plant diversity maintains long-term ecosystem productivity under

412 frequent drought by increasing short-term variation. Ecology 98:2952-2961.

413 Wardle, D.A. 1999. Is "sampling effect" a problem for experiments investigating

414 biodiversity-ecosystem function relationships? Oikos 87:403-407.

415 Wardle, D.A. 2016. Do experiments exploring plant diversity-ecosystem functioning

416 relationships inform how biodiversity loss impacts natural ecosystems? 27:646-653.

417 Weiner, J. and R.P. Freckleton. 2010. Constant final yield. Annual Review of Ecology,

418 Evolution, and Systematics 41:173-192.

419 Westoby, M. 1981. The place of the self-thinning rule in population dynamics. The American $420 \quad$ Naturalist 118:581-587. 
421 Williams, L.J., A. Paquette, J. Cavender-Bares, C. Messier and P.B. Reich. 2017. Spatial

422 complementarity in tree crowns explains overyielding in species mixtures. Nature $423 \quad$ Ecology and Evolution 1:0063.

424 
Figure 1
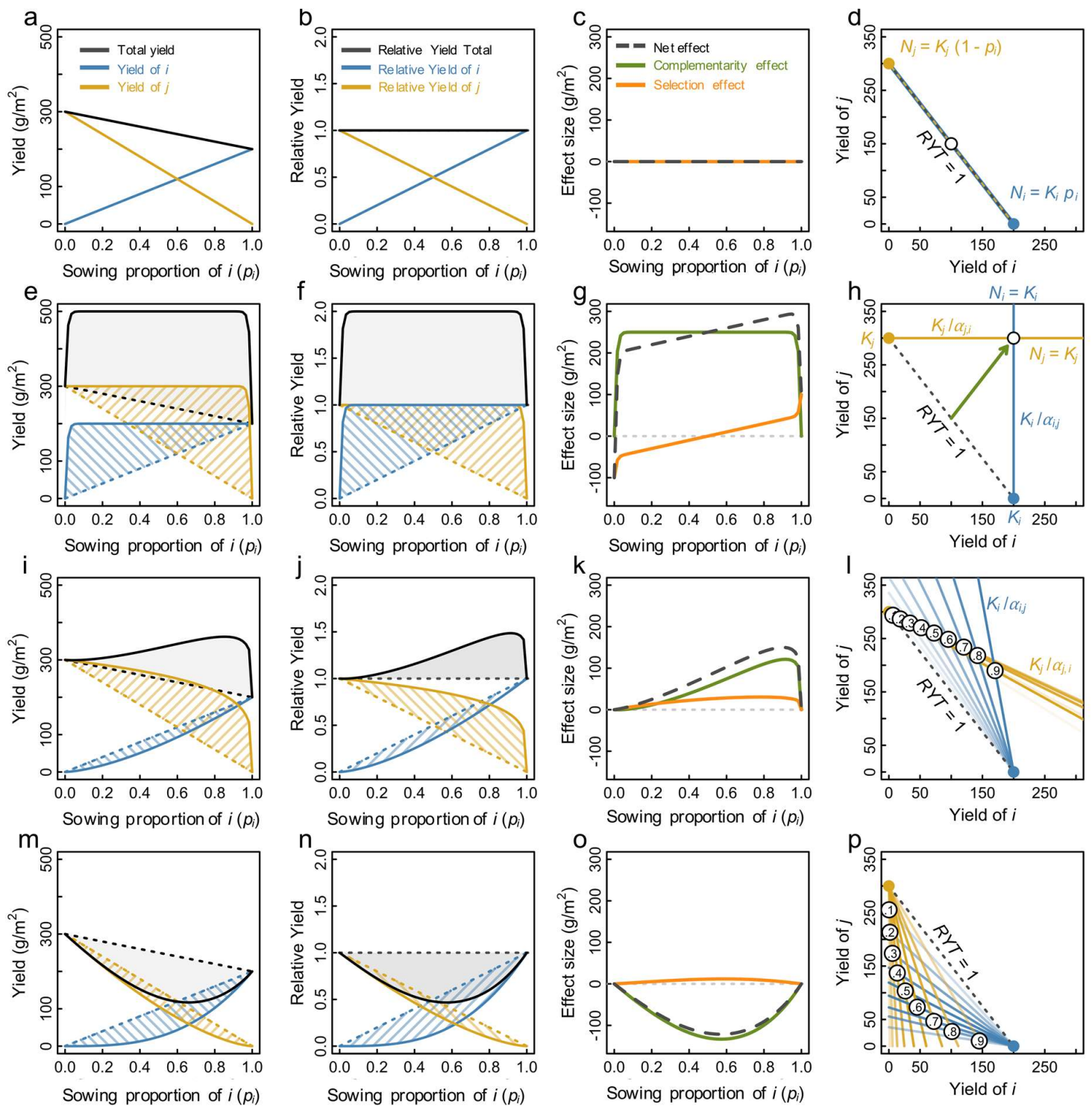

427 Figure 1. Diagram illustrating the classic 'de Wit' style of mixing two species (' $i$ ' and ' $j$ '

428 with monoculture yields of 300 and $200 \mathrm{~g} / \mathrm{m}^{2}$ respectively) along a replacement series design

429 with four different hypothetical scenarios (figure rows). The first scenario (a-d) is the null

430 expectation for biodiversity effects where there are intra- and interspecific interactions are

431 equal and the yield per sown density corresponds to simple mixing of the monoculture yields.

432 The second scenario $(\mathrm{e}-\mathrm{h})$ illustrates when there are only intraspecific and no interspecific

433 interactions, thus species achieve their constant final yield at all except the very lowest 
434 sowing densities, which is an extreme form of a biodiversity effect and the most extreme case

435 of the null expectation of Pillai and Gouhier (2019). The third scenario (i-1) illustrates where

436 the less productive species $i$ (blue) yields less than expected in mixtures, but is

437 overcompensated for by the more productive species $j$ (light-brown) yielding more than

438 expected in mixtures leading to a positive selection and complementarity effect at higher

439 sowing proportions of species $i$ to $j$. The fourth scenario $(\mathrm{m}-\mathrm{p})$ illustrates the case when both

440 species yield less than expected in mixture resulting in a negative complementarity effect

441 (here the less productive species $i$ yields proportionally slightly less than the more productive

442 species $j$, thus, producing a slightly positive selection effect). For each scenario we have

443 illustrated the results in the figure columns of yield (a, e, i, m), relative yield (b, f, j, n), net

444 biodiversity effects (NE, black-dashed line), complementarity effects (green) and selection

445 effects (orange; $\mathrm{c}, \mathrm{g}, \mathrm{k}, \mathrm{o}$ ) and a projection of the outcomes onto species isoclines assuming

446 stable coexistence is achieved at each sowing combination (d, h, l, p, sensu Loreau 2004). For

447 yield and relative-yield panels the dotted lines indicate the expected yields and relative yields

448 under the null hypothesis that the yield of an individual is density independent for both

449 species (first scenario). The shaded region in the yield and relative-yield figures indicates the

450 deviation away from the null expectation. For panels showing NEs, CEs and SEs the lightly

451 dotted grey line highlights $\mathrm{NE}=0$ (no effect). In (d) under the null hypotheses species yield

452 at equilibrium is linearly proportional to its relative sown density. Here the open point simply

453 highlights a 1:1 sowing ratio, but the two species can stably coexist anywhere along the line.

454 In (h) the change in the proportion sown of one species has no effect (no slope) on the yield

455 of the other and species will always achieve an equilibrium yield consistent with their

456 monocultures regardless of their sown proportion. Here the dotted line indicates the expected

457 relative yield total in the absence of any overyielding $(R Y T=1)$ and the green arrow

458 highlights the overyielding effect of the species in mixture. The open point indicates the 
459 yields at which species coexist (i.e. at each sown proportion). In panel (1) species isoclines

460 are shown for each sowing proportion of $i$ from 0.1 to 0.9 in sequential steps of 0.1 (indicated

461 with the open points highlighting the yields at which species coexist, i.e. at each sown

462 proportion). More darkly colored lines indicate a stronger complementarity effect (distance

463 away from $R Y T=1)$. The same is shown in panel (p) as in panel (l) for the situation where

464 species mixing results in under-yielding $(R Y T<1)$.

465 